1.12; respectively) or between the first and fourth week (RR $1.02,95 \%$ CI 0.94 to 1.11 ; RR 0.93 , 95\% CI 0.84 to 1.03 ; respectively). Antibiotic treatment does not led to a lower recurrence (RR 1.28, 95\% CI 0.68 to 2.43 ) in women, but increases the frequency of adverse events reported by sexual partners (RR 2.55, 95\% CI 1.55 to 4.18 ).

Conclusion High quality evidence shows that antibiotic treatment for sexual partners of women with BV, does not increase the rate of clinical or symptomatic improvement and does not led to a lower recurrence rate into the women, but increases the frequency of adverse events reported by sexual partners.

\section{P2.04 TITLE A CLINICAL INVESTIGATION TO IMPROVE REPRODUCTIVE HEALTH SERVICE DELIVERY IN PRIMARY CARE TO REFUGEES FROM BURMA}

${ }^{1}$ Amita Tuteja, ${ }^{1}$ Lena Sanci, ${ }^{2}$ Lester Mascarenhas, ${ }^{3}$ Elisha Riggs, ${ }^{2}$ Lynette 0 Dwyer, ${ }^{3}$ Van Villet Katrina Sangster, ${ }^{2}$ Kim Mcguiness, ${ }^{1}$ Meredith Temple-Smith. ${ }^{\prime}$ Department of General Practice, University of Melbourne, Australia; ${ }^{2}$ Isis Primary Care, Hoppers Crossing, Australia; ${ }^{3}$ Murdoch's Children Research Institute, Royal Children's Hospital, University of Melbourne

10.1136/sextrans-2017-053264.181

Introduction In 2014-2015, Australia granted over 2000 humanitarian visas to people from Burma. During initial refugee health assessments conducted at local health centres, primary care practitioners (PCP) observed that these refugees are quiet, modest and polite; rarely acknowledge sexual health problems and typically answer in the affirmative. Consequently, PCP found it challenging to understand their health needs. Our study aimed to gain insight into how migration affected the reproductive health needs of this group, their ability to access an unfamiliar health system, and to identify elements of a sexual health service model for adaptation to refugee communities.

Methods As we targeted the sensitive needs of a marginalised population we selected qualitative techniques, and conducted 27 semi-structured interviews on sexual health consultations with PCP involved with refugees from Burma. Interviews were audio-recorded and transcribed. Research team members reached consensus on coding, content and thematic analysis and key results.

Results Preliminary analysis suggested six consultation related themes: interpretation, language and euphemisms, culture and beliefs, power dynamics, role of family and low levels of health literacy. Overall, effective communication was the key to successful reproductive health consults. For instance, picture a genital examination with an interpreter on the speaker phone at a distance from the examining couch and practitioner interpreter and patient speaking loudly to communicate the steps of speculum examination. Secondly community leaders often serve as interpreters raising serious privacy concerns in sexual health related problems.

Conclusion The needs of the refugee patient cannot always be ascertained through the biomedical lens. PCP need assistance to contextualise 'behind the scene' communication dynamics involved in sexual health consults. Humanising perspectives can assist in overcoming barriers. Patient's must be given a choice of interpreter in sexual and reproductive health consultations in refugee settings.

\section{P2.05 A SILENT EPIDEMIC: THE PREVALENCE, INCIDENCE AND PERSISTENCE OF MYCOPLASMA GENITALIUM IN YOUNG ASYMPTOMATIC WOMEN IN THE UNITED STATES}

${ }^{1}$ Arlene C Seña, ${ }^{2}$ Jeanette Y Lee, ${ }^{3}$ Jane Schwebke, ${ }^{4}$ Susan S Philip, ${ }^{5}$ Harold C Weisenfeld, ${ }^{6}$ Anne M Rompalo, ${ }^{7}$ Robert I Cook, ${ }^{1}$ Marcia M Hobbs. ${ }^{1}$ Department of Medicine, University of North Carolina at Chapel Hill, USA; ${ }^{2}$ Department of Biostatistics, University of Arkansas for Medical Sciences, USA; ${ }^{3}$ Department of Medicine, University of Alabama at Birmingham, USA; ${ }^{4}$ San Francisco Department of Public Health, USA; ${ }^{5}$ Department of Obstetrics and Gynaecology, Magee-Women's Hospital of University of Pittsburgh, USA; ${ }^{6}$ Department of Medicine, Johns Hopkins University School of Medicine, USA; 'Departments of Epidemiology and Medicine, University of Florida, USA

10.1136/sextrans-2017-053264.182

Introduction: Mycoplasma genitalium (MG) is an emerging sexually transmitted infection (STI) associated with cervicitis, pelvic inflammatory disease, and adverse pregnancy outcomes in women, yet little is known regarding its natural history. We conducted a secondary analysis of specimens collected from young women enrolled in a multi-centre study of asymptomatic bacterial vaginosis (BV) in order to determine the natural history of MG and associated factors with infection.

Methods Sexually active women aged 15-25 years were recruited from 10 US clinical sites. Eligible women had asymptomatic BV at baseline, and $>/=2$ STI risk factors. Selfcollected vaginal swabs were collected at enrollment, and by home-based testing at 2, 4, 6, 8, 10 and 12 months. MG nucleic acid amplification testing was performed using a transcription mediated assay (Hologic Inc, San Diego, California). Prevalent, incident and persistent MG were estimated with 95\% confidence intervals (CI). Univariate analyses and logistic regression modelling were performed to assess associations between participants' baseline demographic, sexual, and clinical characteristics with prevalent MG infection.

Results Specimens were tested for MG from 1365 predominantly Black (85.4\%) women. At baseline, 233 women were MG+ (prevalence 20.5\% [95\% CI: 18.2-22.9]); among 204 participants with follow-up specimens, 42 (20.6\%) had persistent $\mathrm{MG}$, remaining $\mathrm{MG}+$ on all follow-up testing. Among 801 women who were MG negative at baseline with followup testing, 220 had at least one subsequent $\mathrm{MG}+$ test for an incidence of $27.5 \%$ (95\% CI: 24.4-30.7). Black race (adjusted odds ratio (AOR) 1.92, 95\% CI: 1.09-3.38) and younger (1521 years) age (AOR 1.40, 95\% CI: 1.03-1.91) were significantly associated with prevalent MG infection.

Conclusions We identified high rates of prevalent, incident, and persistent MG infections among sexually active young women followed over 12 months. As national programs consider the impact of MG as an STI, the implications of asymptomatic infections should be considered among at-risk populations.

\section{P2.06 TRENDS IN CD4 COUNT AND WHO STAGING AMONG NEWLY DIAGNOSED HIV PATIENTS ATTENDING AN ANTI-RETROVIRAL THERAPY CENTRE IN TERTIARY CARE HOSPITAL}

Basavaprabhu Achappa. Kasturba Medical College, affiliated to Manipal University, India

10.1136/sextrans-2017-053264.183

Introduction Patients diagnosed with HIV infection late in course of disease are usually more severely 